\title{
Magneto-elastic effect in non-Newtonian ferrofluid droplets impacting on superhydrophobic surfaces
}

\author{
Gudlavalleti V V S Vara Prasad a, Purbarun Dhar ${ }^{b, *, 1}$ and Devranjan Samanta ${ }^{a, *, 2}$ \\ a Department of Mechanical Engineering, Indian Institute of Technology Ropar, \\ Rupnagar-140001, Punjab, India \\ ${ }^{\mathrm{b}}$ Department of Mechanical Engineering, Indian Institute of Technology Kharagpur, \\ Kharagpur-721302, West Bengal, India
}

*Corresponding authors:

1E-mail: purbarun@mech.iitkgp.ac.in; purbarun.iit@gmail.com

${ }^{1}$ Tel: +91-3222-28-2938

2E-mail: devranjan.samanta@iitrpr.ac.in

${ }^{2}$ Tel: +91-1881-24-2109

\section{Supplementary information}

Table. S1: Surface tension values of the non-Newtonian ferrofluid droplets

\begin{tabular}{|c|c|c|}
\hline Base fluid & $\mathrm{Fe}_{3} \mathrm{O}_{4}$ particles & Surface tension $(\mathrm{mN} / \mathrm{m})$ \\
\hline \multirow[t]{2}{*}{ P0 } & F2.5 & 72.6 \\
\hline & F5 & 75.4 \\
\hline \multirow[t]{2}{*}{ P5 } & F2.5 & 74 \\
\hline & F5 & 76 \\
\hline \multirow[t]{2}{*}{ P10 } & F2.5 & 77.5 \\
\hline & F5 & 79 \\
\hline
\end{tabular}




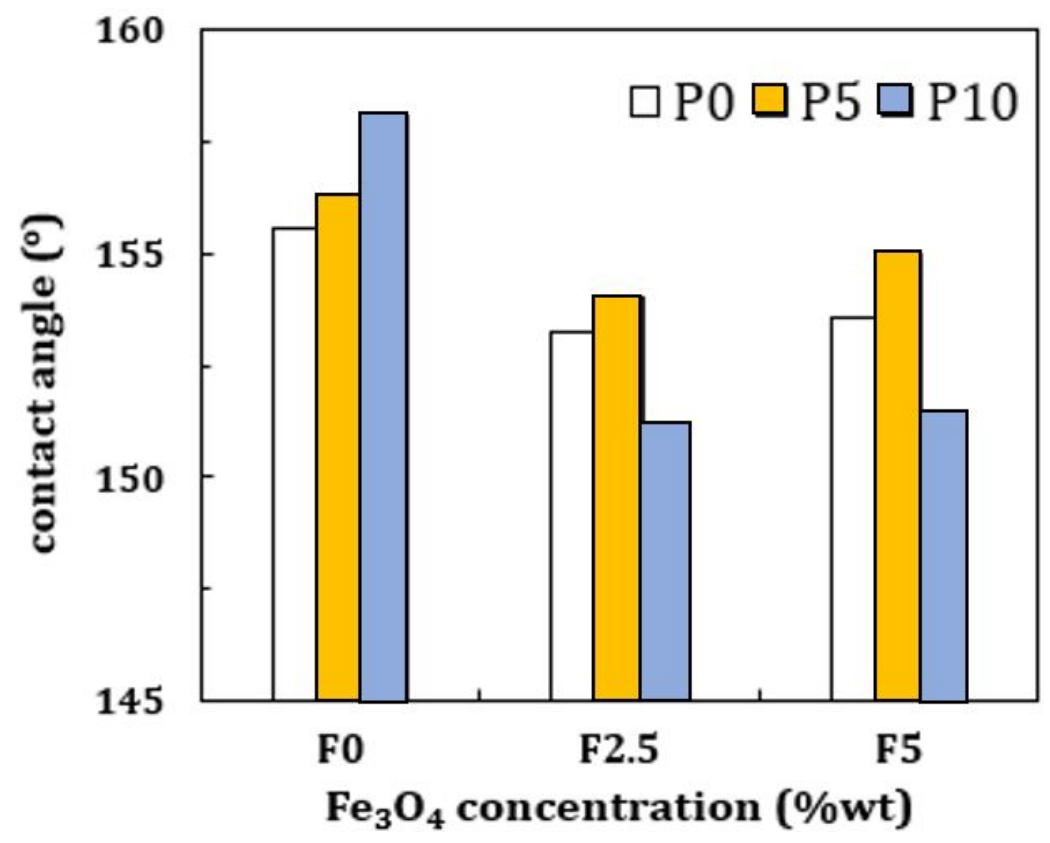

Fig. S1: Static equilibrium contact angles of different non-Newtonian ferrofluid droplets on spray coated superhydrophobic surface.

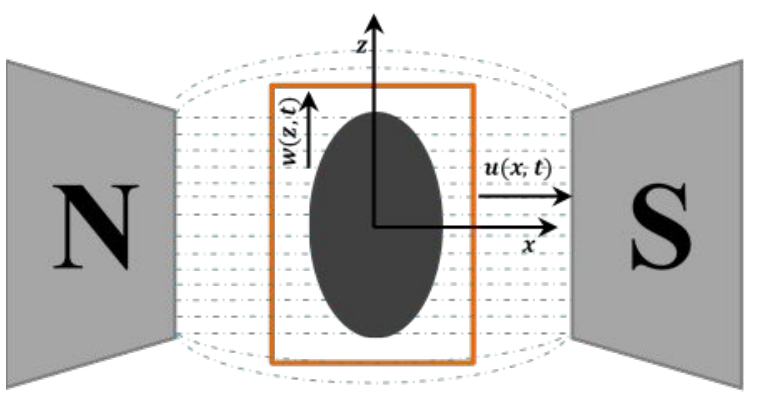

(i)

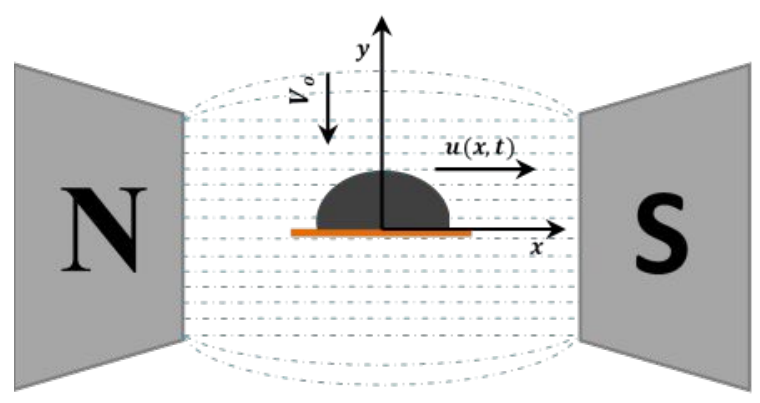

(ii)

Fig. S2: (i) top view and (ii) front view of the ferrofluid droplet under magnetic field. $\mathrm{N}$ and $S$ represent the north and south poles of the electromagnet. 


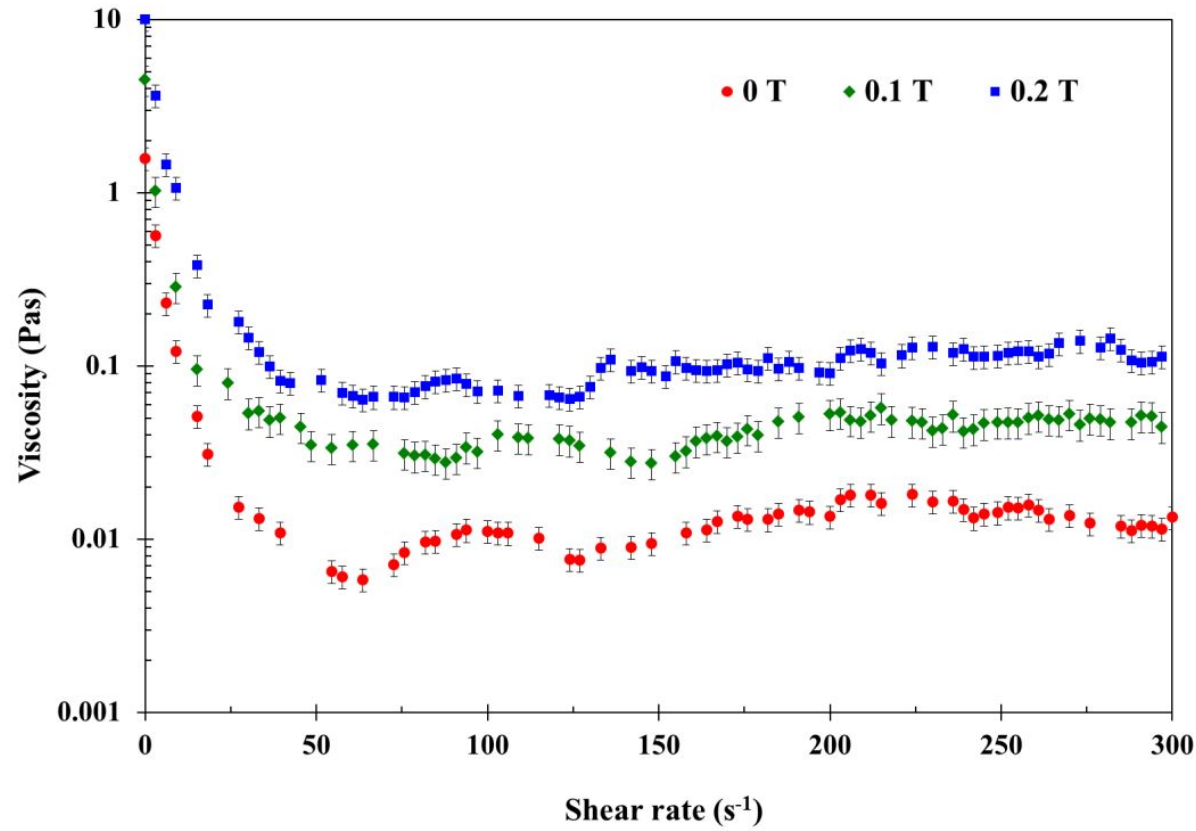

Fig S3: Rheological response of the non-Newtonian ferrofluid (P10-F5) in the presence of magnetic field. The sample with the highest polymer and $\mathrm{Fe}_{3} \mathrm{O}_{4}$ content has been chosen to explain the rheology in the context of the most viscous sample. It is noted that the nonNewtonian character of the ferrofluids is retained only up to shear rates of $\sim 50-100 \mathrm{~s}^{-1}$. This behaviour is also conserved in case of the magnetorheology. Beyond this regime, the fluids show nearly Newtonian behaviour, albeit with enhanced Newtonian viscosity due to the magnetic field.

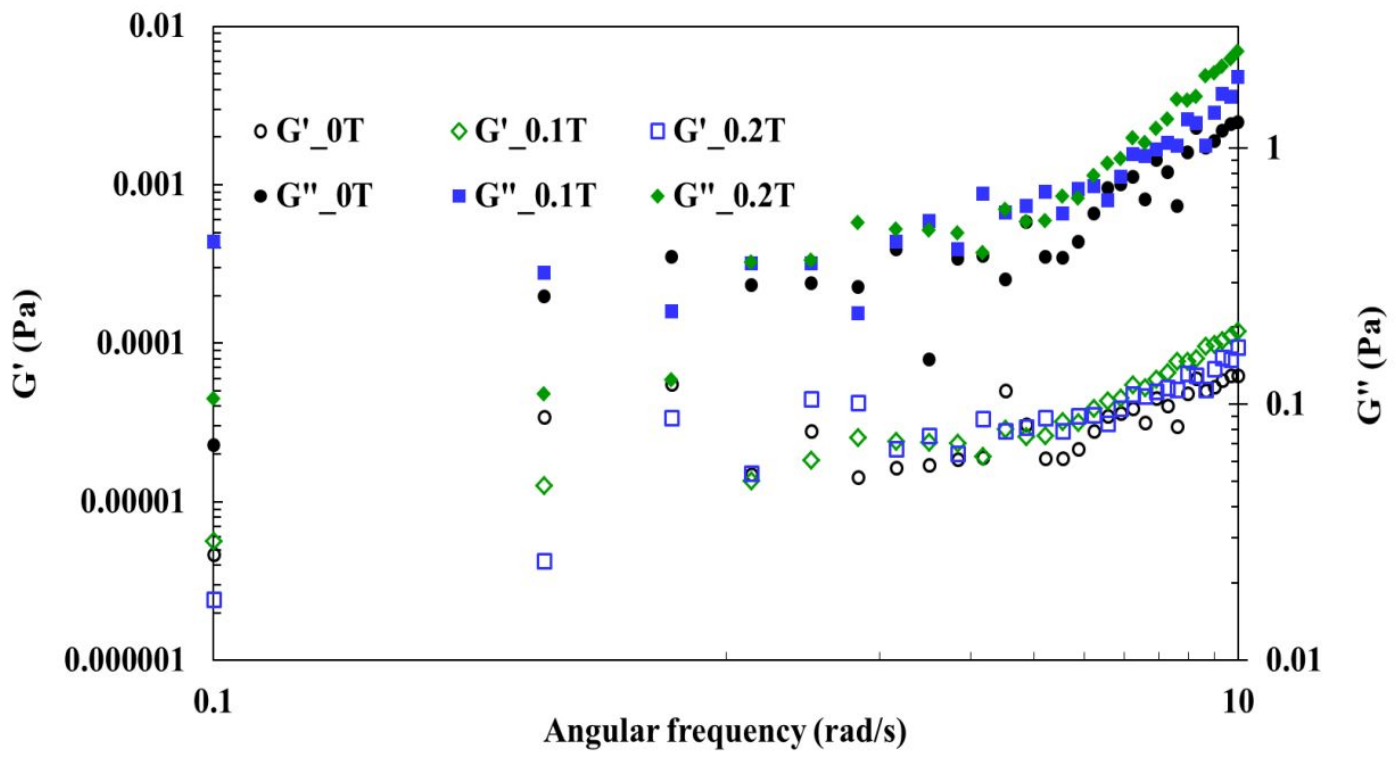

Fig S4: Viscoelastic response of the non-Newtonian ferrofluid (P10-F2.5) due to increasing oscillatory angular frequency at $1 \%$ oscillatory strain amplitude. A single case has been illustrated for representation purpose. The oscillatory frequency $(\omega)$ dependent values of $G^{\prime}$ and $G$ " are used to determine the frequency dependent complex viscosity as $\boldsymbol{\eta}_{\boldsymbol{C}}=\boldsymbol{\omega}^{-\mathbf{1}}$ $\sqrt{\boldsymbol{G}^{\mathbf{2}}+\boldsymbol{G}^{\mathbf{\prime \prime}}}$, which is further used to estimate the relaxation time scales for the nonNewtonian fluids. 

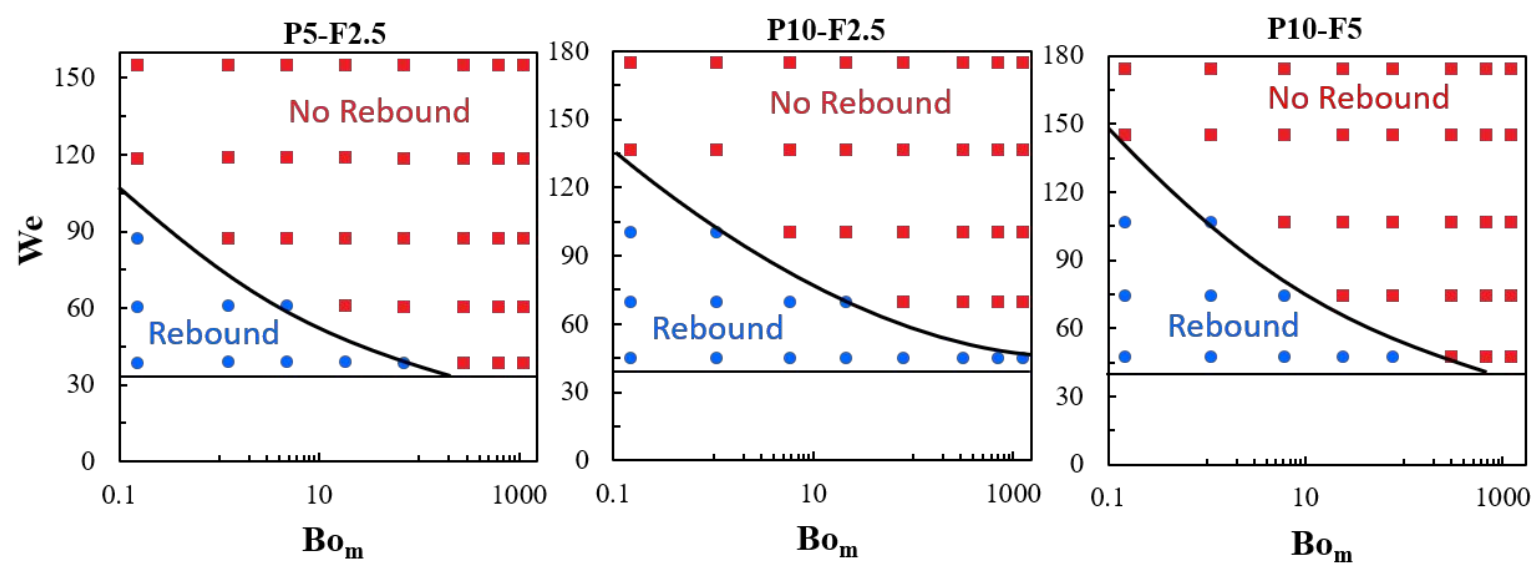

Fig. S5 Demarcation of "rebound (combined RI and RII in main paper)" and "no rebound" cases of impacting non-Newtonian ferrofluid droplets in external magnetic field by changing both polymer concentration and magnetic nanoparticle concentration.

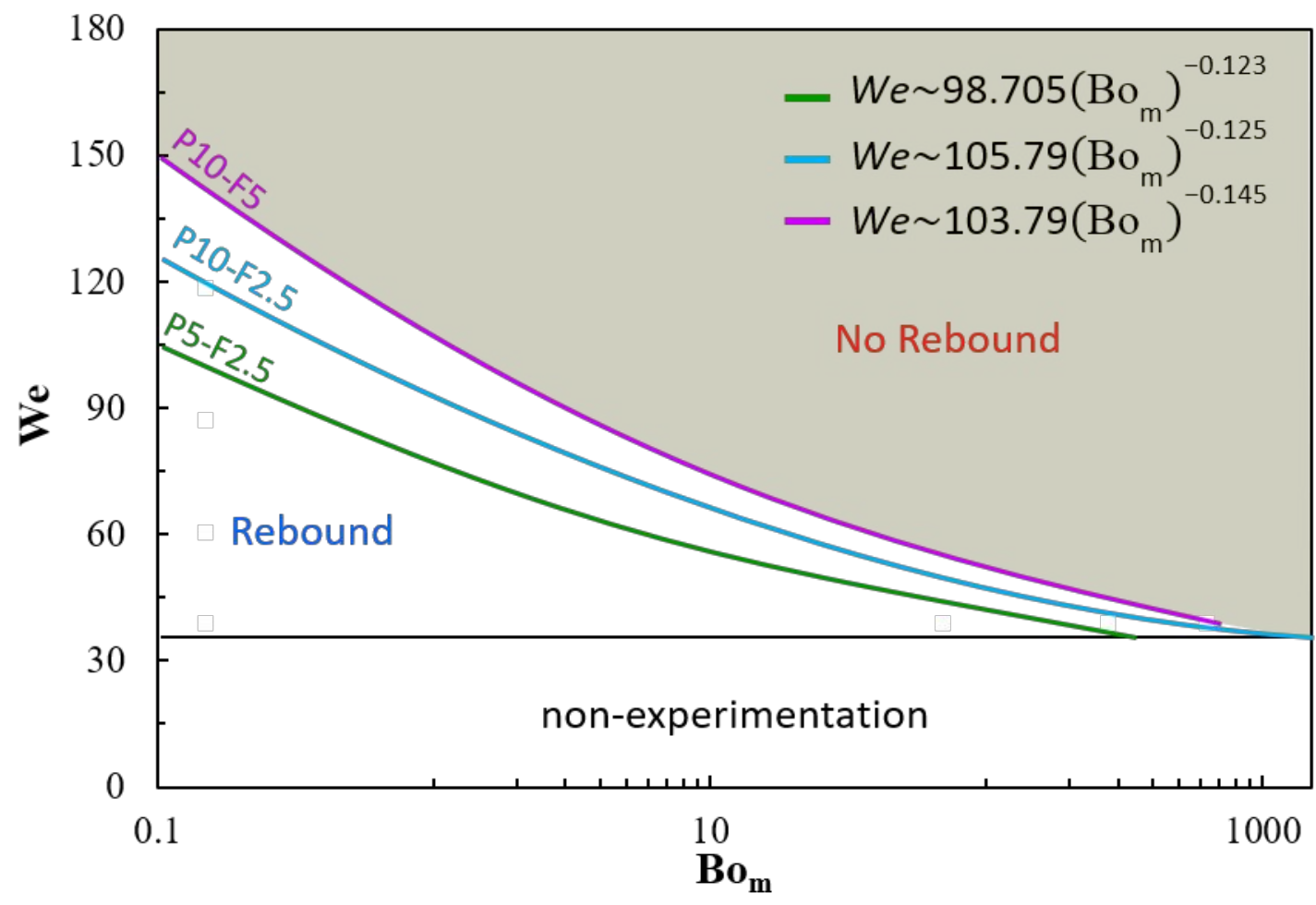

Fig. S6 Scaling relationship between critical $W e$ and $B o_{m}$ to demarcate the "rebound" and "no rebound" behaviours of impacting non-Newtonian ferrofluid droplet for different concentrations of both polymer and magnetic nano particles. 


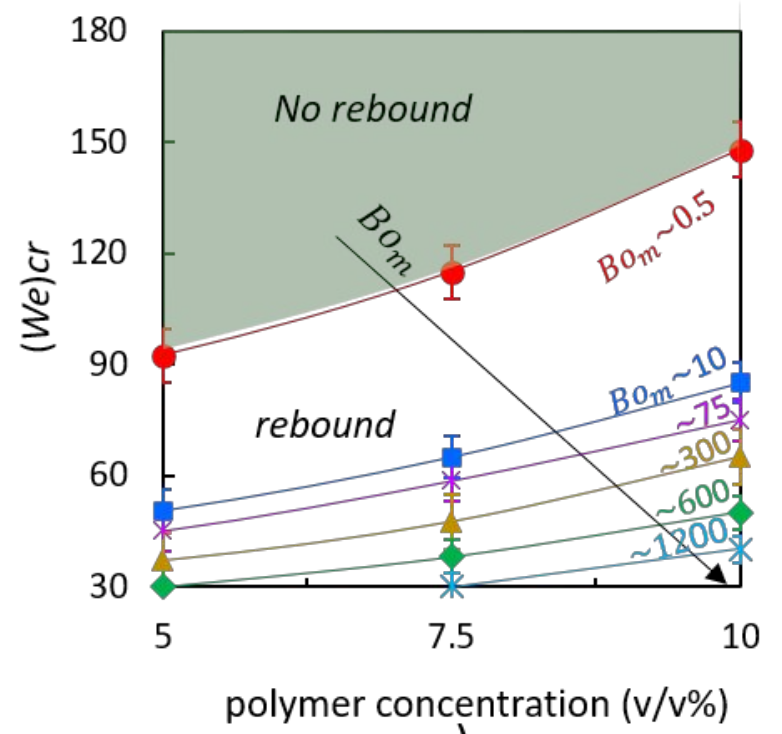

a)

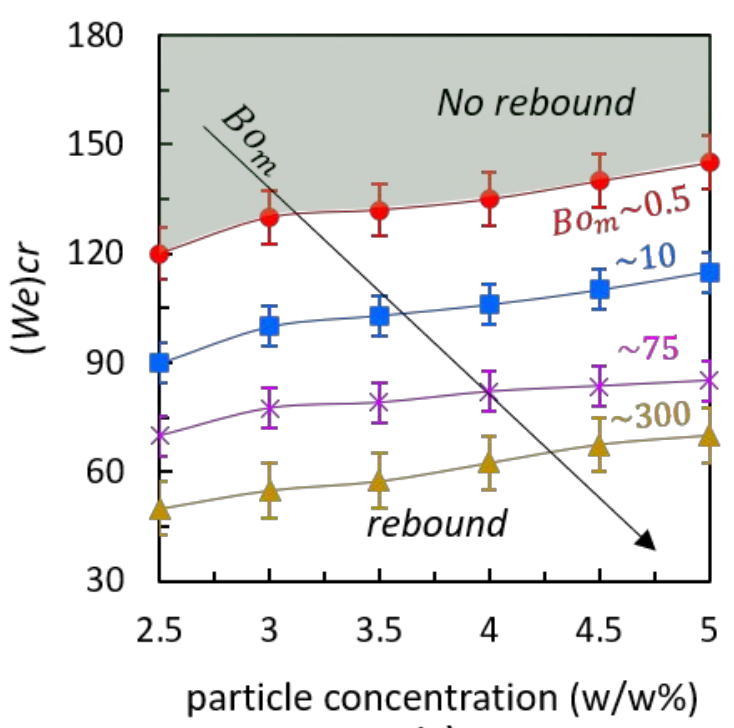

b)

Fig. S7 (a) and (b) shows the critical We (across which We ferrofluid droplet "rebound" behaviour turns into "no rebound" and vice versa) over different polymer concentrations and magnetic nanoparticles concentrations respectively. 